PHOTOMETRIC, SPECTROSCOPIC AND 21-cm LINE INVESTIGATION OF SELECTED UV-EXCESS KISO AND CLUMPY GALAXIES

L. Bottinel1i ${ }^{1}$, L. Gouguenheim ${ }^{1}$, M. Hamabe ${ }^{2}$, J. Heidmann ${ }^{1}$, H. Maehara ${ }^{2}$, B. Takase

${ }^{1}$ Paris Observatory, Meudon 92195, France

${ }^{2}$ Tokyo Astronomical Observatory, Mitaka, Tokyo 181, Japan

${ }^{3}$ Kokugakuin University, Shibuya, Tokyo 150, Japan

\title{
1. PHOTOMETRY
}

A survey of Kiso UV-excess galaxies (KUGs) has been made using the 105$\mathrm{cm}$ Kiso Schmidt telescope. We selected three galaxies of Ic-type (i.e. irregulars with clumpy H II regions). KUGs $1618+373,1624+404$ and $1626+$ 413, and observed them together with a clumpy irregular galaxy Mrk 297 to clarify their star formation activity (Maehara et al. 1986).

Photometric observations in both $b$ (blue) and $r$ (red) bands were made with the PIRAMIG camera attached to the 2-m Pic-du-Midi telescope Photographs were measured and processed using the PDS micro-densitometer and the superminicomputer FACOM S-3500 at Kiso by means of the standard method of surface photometry of galaxies. The isophotometric contour of KUG $1626+413$ in the blue band is given in Figure 1 .

Blue isophotes show a number of clumps scattered over the galaxies. Absolute $b$ magnitudes of these clumps range from -10 to -18 , much brighter than galactic HII regions. The average color of peripheral clumps is bluer than that of the central ones.

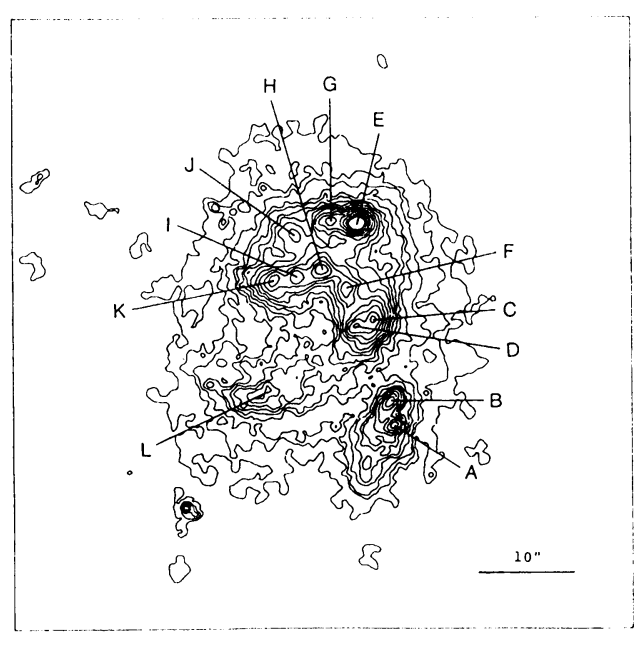

Fig. 1. Blue isophote of KUG $1626+413$. 


\section{SPECTROSCOPY}

Low-resolution (237 $\mathrm{A} \mathrm{m}^{-1}$ ) spectrograms were obtained with the Cassegrain image-intensifier spectrograph attached to the $188-\mathrm{cm}$ Okayama telescope. These galaxies have conspicuous emission lines similar to those of H II regions. From their radial velocities, the three KUGs are located at $\sim 120 \mathrm{Mpc}$ from us (assuming $\mathrm{H}_{0}=75 \mathrm{~km} \mathrm{~s}^{-1} \mathrm{Mpc}^{-1}$ ), and their absolute magnitudes range from -20 to -21 in the blue band.

A diagram of 1 ine-intensity ratios between [ 0 III] $\lambda 5007 / \mathrm{H} \beta$ and [ $\left.\mathrm{NI}_{2}\right] \lambda 6584 / \mathrm{H} \alpha$ is very efficient for the classification of emissionline objects. The ratios derived from the present samples reveal that al1 of them are located in the HII region domain. That is, the blue clumps of these galaxies are HII regions on larger scales. The two bright clumps of KUG $1618+378$ are similar to low-excited nuclear HII regions, while the others are high1y-excited disk ones.

\section{21-cm LINE AND IR PROPERTIES}

Observations of the $21-\mathrm{cm}$ hydrogen 1 ine were made with the 1024-channel autocorrelation spectrometer of the Nançay $300-\mathrm{m}$ radio telescope, which has a half power beam width of $4^{\prime}$ (EW) $\times 22^{\prime}$ (NS). It is shown that the clumpy irregular galaxy Mrk 297 and the three KUGs have HI contents up to 7 times larger than classical irregulars, and also luminosities from 4 to 13 times larger.

All the sample galaxies were detected by the IRAS from 25 to $100 \mu \mathrm{m}$. Figure 2 gives the IR flux densities, reduced to a common distance of $100 \mathrm{Mpc}$ from us. They are from 3 to 10 times brighter in IR than the strong starburst galaxy M82. It is suggested that the observed IR fluxes are due to warm dust ( $\sim 40 \mathrm{~K}$ ) associated with $\mathrm{H}$ II regions and molecular clouds, which reradiate the radiation of newly born stars.

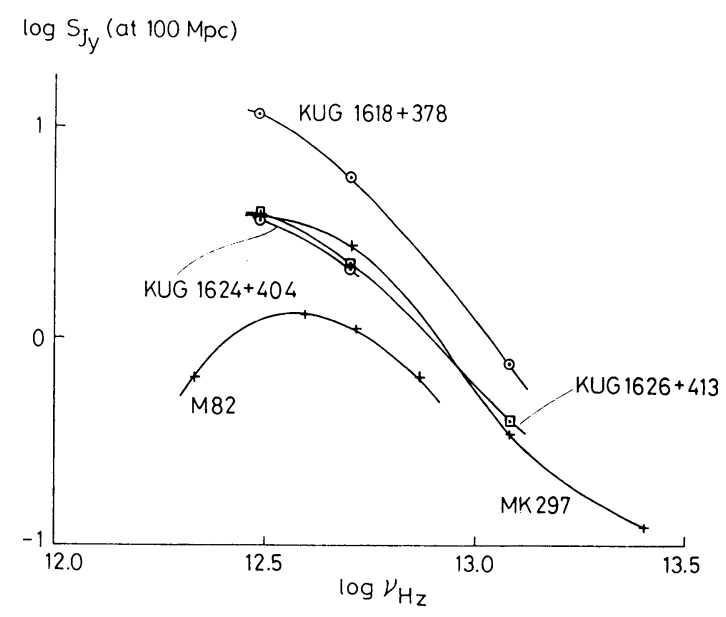

Fig. 2. IR flux densities of the objects together with M82. 


\section{CONCLUSIONS}

In conclusion, the present analysis reveals the following facts for the three Ic-type KUGs $1618+378,1624+404$, and $1626+413$ and the clumpy irregular galaxy Mrk 297:

(1) These galaxies contain several to more than ten bright blue clumps which are scattered out to the peripheral regions.

(2) The excitation mechanisms of these clumps are similar to galactic

H II regions, but the scales and brightnesses are much larger.

(3) KUG $1626+413$ probably belongs to the clumpy irregular type, and the two other KUGs may be spiral galaxies much perturbated by active star formation. Mrk 297 is confirmed as an active starburst galaxy.

(4) From the FIR fluxes, they have a star formation rate 3 to 10 times larger than the starburst galaxy M82.

\section{REFERENCES}

Maehara, H., Hamabe, M., Bottine11i, L., Gouguenheim, L., Heidmann, J., and Takase, B.: 1986, in preparation.

NUMERICAL SIMULATIONS OF STAR FORMATION BURSTS INDUCED BY THE GALAXY-GALAXY INTERACTION

Masafumi Noguchi

Tokyo Astronomical Observatory, Mitaka, Tokyo 181, Japan

Shiro Ishibashi

Department of Astronomy, University of Tokyo, Tokyo 113, Japan

The galaxy-galaxy interaction has been proposed as a possible triggering mechanism of the star formation bursts in some galaxies (e.g. Larson and Tinsley 1978). To investigate the nature of star formation bursts triggered by interaction we have numerically simulated close encounters between disk galaxies, taking the star formation process into account (see Noguchi and Ishibashi 1986 for details). We used the cloudparticle model, in which gas clouds move as test particles in the gravitational field of the galaxies. When two clouds collide with each other, an $O B-s t a r$ is formed. The cloud system loses its kinetic energy by inelastic cloud-cloud collisions. The supernova explosion which follows the formation of an OB-star provides kinetic energy to the nearby clouds.

Prior to the encounter simulation, an equilibrium model of a disk galaxy (with a mass $=1.8 \times 10^{11} \mathrm{M}_{\odot}$ and a radius $=20 \mathrm{kpc}$ ) is made in which the star formation rate (SFR, i.e. the number of OB-stars formed in each time step) is nearly constant. This model represents an isolated 